\title{
Comparação da variação da actividade neuromuscular, da creatina quinase e da força isométrica máxima voluntária entre dois protocolos exaustivos e inabituais
}

\author{
T.M.Barbosa ${ }^{1}$ \\ P.M. Magalhães ${ }^{1}$ \\ V.P. Lopes ${ }^{1}$ \\ M. Neuparth ${ }^{2}$ \\ J.A. Duarte ${ }^{2}$
}

https://doi.org/10.5628/rpcd.03.01.07

\section{RESUMO}

O objectivo deste trabalho foi comparar a variação da actividade neuromuscular, da força isométrica máxima voluntária e da criatina quinase entre um protocolo exaustivo de exercícios de contracções concêntricas e de contracções excêntricas. Foram avaliados 15 sujeitos do sexo masculino saudáveis e não treinados. 8 realizaram exclusivamente um exercício com contracções concêntricas e 7 realizaram exclusivamente um exercício com contracções excêntricas. Foi avaliada a amplitude do sinal electromiográfico, a mediana da frequência, a força isométrica máxima voluntária e as concentrações de creatina quinase. Estas variáveis foram analisadas imediatamente antes do protocolo, imediatamente após o protocolo, 1 hora, 3 horas, 24 horas, 48 horas e 72 horas após o término do protocolo. A mediana de frequência apresentou valores significativamente superiores durante o exercício de contracções concêntricas do que durante o exercício de contracções excêntricas 1 hora, 3 horas e 24 horas após o protocolo, mas foi significativamente inferior às 72 horas. A força isométrica máxima voluntária foi significativamente superior ao realizar contracções concêntricas do que excêntricas em todos os momentos de avaliações.

A creatina quinase foi significativamente superior nas contracções excêntricas do que nas concêntricas 48 horas após o término do protocolo.

Palavras-chave: actividade neuromuscular, força isométrica, creatina quinase, contracção concêntrica, contracção excêntrica.
${ }^{1}$ Escola Superior de Educação, Instituto Politécnico de Bragança, Portugal

${ }^{2}$ Faculdade de Ciências do Desporto e de Educação Física, Universidade do Porto, Portugal

\begin{abstract}
Comparison of neuromuscular activity, creatine kinase and maximal isometric voluntary force variation between two exhaustive and not habitual protocols

The aim of this study was to compare the neuromuscular activity, the maximal voluntary isometric force and the creatine kinase variation between an exhaustive concentric contraction protocol and an exhaustive eccentric contraction protocol. 8 healthy and detrained males made concentric contractions and 7 made eccentric contractions protocol. The parameters evaluated were the electromyographic amplitude, the median frequency, the maximal voluntary isometric force and the creatine kinase. All parameters were measured before the protocol, immediately after the protocol, 1 hour, 3 hours, 24 hours, 48 hours and 72 hours after the protocol. The median frequency presented values significantly higher on the concentric contraction group than in the eccentric contraction group on the 1st, 3rd and 24th hour but, significantly lower on the 72th hour. The maximal voluntary isometric force was significantly higher on the concentric contraction group than the eccentric contraction group in all moments. The creatine kinase was significantly higher on the eccentric contraction group than in the concentric contraction group on the 48 th hour.
\end{abstract}

Key Words: neuromuscular activity, isometric force, creatine kinase, concentric, eccentric 


\section{INTRODUÇÃO}

O exercício físico inabitual e exaustivo induz alterações estruturais, ultra-estruturais e bioquímicas de carácter focalizado e reversível $(7,19,20,23)$. Estas alterações são características de uma patologia aguda denominada por Miopatia do Exercício (ME).

Existem evidências de que a exuberância dos sinais e sintomas associados à ME está dependente do tipo de contracções (concêntricas vs. excêntricas), da intensidade, da duração $(7,20,34)$ e de factores relacionados com o próprio indivíduo, como a idade e o nível de treino para a tarefa proposta (34). Com efeito, encontram-se descritas na literatura diversas evidências indirectas de lesão muscular, que parecem associar a elevada percentagem de contracções excêntricas a uma maior evidência da sintomatologia associada à ME. Nomeadamente: (i) diminuições mais acentuadas da força isométrica máxima voluntária $(18,26,27)$; (ii) aumentos significativamente superiores da sensação de desconforto muscular (1, 4, 12, 23); (iii) aumentos significativamente superiores do perímetro braquial $(5,11,28)$ e; (iv) aumentos significativamente superiores das concentrações plasmáticas da creatina quinase $(2,5,8,26)$. Efectivamente, a creatina quinase é o indicador bioquímico mais utilizado na literatura como indicador da ocorrência de lesão muscular $(20,35)$. A sua presença acentuada no plasma sanguíneo sugere a existência de uma elevada agressão sobre as fibras musculares, com lesão dos miofilamentos, do sarcolema e de organelos sub-celulares (7). Por outro lado, autores como Lieber e Fridén (16) referem a maior tensão exercida sobre as fibras musculares pelas contracções excêntricas em comparação com o trabalho isométrico ou concêntrico, num estudo realizado com ratos. Ou seja, parece existir já alguma produção científica no sentido de tentar compreender os processos subjacentes à patologia em causa, em função do tipo de tensão muscular produzida.

Todavia, as repercussões deste fenómeno a nível neuromuscular não parecem estar completamente esclarecidas $(6,9,21,22,25)$. E isto não obstante Sbriccolo et al. (33) considerarem que a electromiografia é uma das metodologias mais eficientes na detecção precoce das consequências das contracções do tipo excêntrico na patologia em estudo.
Foi objectivo deste trabalho conhecer as repercussões que se verificam na actividade neuromuscular após a realização de diferentes protocolos de exercício físico (contracções concêntricas vs. contracções excêntricas) com carácter inabitual e exaustivo.

\section{MATERIAIS E MÉTODOS}

\section{Amostra}

A amostra foi constituída por 15 sujeitos do sexo masculino clinicamente saudáveis e não treinados $(21.0 \pm 2.0$ anos de idade, $170.2 \pm 5.8 \mathrm{~cm}$ de estatura e $67.513 \pm 8.010 \mathrm{Kg}$ de massa corporal). A todos os indivíduos, antes de darem o seu consentimento para participarem no estudo, foi explicado o objectivo do mesmo e os procedimentos a que estariam sujeitos. Os 15 sujeitos foram divididos aleatoriamente em dois grupos. 8 sujeitos constituíram um grupo que realizou exclusivamente um exercício de contraç̧ões do tipo concêntrico (CC) e os restantes 7 exclusivamente um exercício de contracções excêntricas (CE). De forma a assegurar o carácter inabitual do exercício proposto, foi solicitado aos elementos da amostra que não realizassem qualquer tipo de actividade física nas duas semanas anteriores e durante o tempo de duração do protocolo experimental.

\section{Protocolo experimental}

O protocolo consistiu na execução de um exercício de elevação ou de abaixamento de um haltere com uma resistência de $70 \%$ de $1 \mathrm{RM}$, respectivamente através da flexão ou da extensão do antebraço sobre o braço até à exaustão, com os dois membros superiores em simultâneo. O grupo CC realizou apenas contracções concêntricas e o grupo CE unicamente contracções excêntricas. Para tal, cada indivíduo foi auxiliado por dois sujeitos colocados nas extremidades do haltere que realizavam o movimento oposto. O exercício foi executado na posição de sentado, com os dois braços encostados na sua região posterior a um apoio existente para o efeito. Desta forma, foi possível eliminar hipotéticas contracções parasitas, não directamente envolvidas na execução do exercício. A velocidade de execução foi controlada através da marcação por um metrónomo, sendo realizada uma repetição em cada dois segundos.

Quando o indivíduo não fosse capaz de acompanhar 
o ritmo estipulado, era-lhe proporcionado um intervalo de repouso de 30 segundos. O protocolo era dado como terminado quando o sujeito demonstrasse uma manifesta incapacidade de realizar mais de duas repetições, acompanhando o ritmo indicado, durante três séries consecutivas.

\section{Parâmetros estudados}

Todos os parâmetros foram avaliados antes da aplicação do protocolo experimental ( $\mathrm{Tr}$ ), imediatamente após o seu término (T0), bem como após 1 hora (T1), após 3 horas (T3), após 24 horas (T24), após 48 horas (T48) e após 72 horas (T72) o fim do protocolo. Foi colocado um eléctrodo bipolar (TSD 150A da Biopac Systems Inc.) no bicípete braquial de um dos membros superiores. Antes da sua aplicação, a pele nessa área foi preparada e limpa. Não obstante as limitações que acarreta, mas tentando a atenuar a variabilidade na localização do eléctrodo nos diversos momentos de avaliação, realizaram-se pequenas tatuagens temporárias no local onde este seria colocado, como sugerem Hakkinen e Komi (13) e Hakkinen et al. (14). O eléctrodo de referência foi colocado no acrómio do mesmo lado do bicípete braquial analisado. Utilizou-se um amplificador diferencial com uma impedância de entrada de 2 Megaohms, um ganho de 1000, uma banda passante entre os 15 e os $450 \mathrm{~Hz}$ e uma rejeição do modo comum do amplificador de $90 \mathrm{~dB}$. O sinal electromiográfico foi tratado no aplicativo AcqKnowledge v. 3.5.3. da Biopac Systems Inc. Determinou-se a amplitude do sinal electromiográfico (aEMG) após o sinal ter sido rectificado. Foi calculada a transformada de Fourier, obtendo-se a mediana de frequência (MF).

Simultaneamente com a recolha do sinal electromiográfico também foi avaliada a força isométrica máxima voluntária (FIMV), através de um dinamómetro TSD121C da Biopac Systems Inc, durante 5 segundos. A recolha deste parâmetro fez-se com os indivíduos sentados, com a região posterior do braço encostado no apoio e fazendo um ângulo relativo de aproximadamente $90^{\circ}$ com o antebraço, estando este na horizontal. O sujeito realizava uma contracção isométrica, procurando elevar uma barra que se encontrava fixa nas suas extremidades por correntes, as quais estavam presas no dinamómetro e este a um apoio no solo. Foi recolhido sangue capilar e avaliada a actividade plasmática da creatina quinase (CK) através de um Refreton Analyser da Boehringer Mannheim. Todos os dados foram relativizados com base nos valores recolhidos antes da aplicação do protocolo, permitindo conhecer a percentagem de variação de cada variável.

\section{Tratamento estatístico}

Para o tratamento estatístico dos dados foram determinadas as estatísticas descritivas média e desvio padrão. Para a comparação dos parâmetros estudados entre momentos, em cada grupo, utilizou-se a ANOVA de medidas repetidas. A comparação entre grupos, em cada momento de avaliação, foi realizada a partir do teste t. Em todos os procedimentos estatísticos foi adoptado um nível de significância de 5\% $(\mathrm{p}<0.05)$.

\section{RESULTADOS}

Existem diversas variáveis fisiológicas que são habitualmente utilizadas enquanto indicadores indirectos do nível de lesão induzida pelo exercício físico exaustivo e/ou inabitual. De entre essas variáveis a FIMV e a CK são duas das mais utilizadas $(3,20)$. No presente estudo estes dois parâmetros foram adoptados com o intuito de determinar se o protocolo aplicado permitiu submeter os sujeitos à patologia indicada.

A Figura 1 apresenta as variações dos indicadores de lesão muscular estudados, entre os diferentes momentos de avaliação em cada grupo. No grupo CC, a FIMV apresentou valores significativamente inferiores relativamente a $\mathrm{Tr}$ em $\mathrm{T} 0[\mathrm{~F}(1 ; 7)=$ 58.393, $\mathrm{p}=0.0001]$, em T1 $[\mathrm{F}(1 ; 7)=41.475$, $\mathrm{p}=0.0004]$, em T3 $[\mathrm{F}(1 ; 7)=28.095, \mathrm{p}=0.0011]$, em $\mathrm{T} 24[\mathrm{~F}(1 ; 7)=12.252, \mathrm{p}=0.0100]$ e em $\mathrm{T} 48[\mathrm{~F}(1 ; 7)=6.149, \mathrm{p}=0.0422]$. No grupo CE, os valores foram significativamente inferiores relativamente a Tr em T0 $[\mathrm{F}(1 ; 6)=273.970, \mathrm{p}<0.0001]$, em T1 $[\mathrm{F}(1 ; 6)=393.262, \mathrm{p}<0.0001]$, em T3 $[\mathrm{F}(1 ; 6)=227.365, \mathrm{p}<0.0011]$, em T24

$[\mathrm{F}(1 ; 6)=530.934, \mathrm{p}<0.0001]$, em $\mathrm{T} 48[$ $\mathrm{F}(1 ; 7)=903.942, \mathrm{p}<0.0001]$ e em T72 $[\mathrm{F}(1 ; 6)=404.571, \mathrm{p}<0.0001]$.

A CK, no grupo CC, apresentou valores de actividade plasmática significativamente superiores relativamente a Tr em T0 $[F(1 ; 7)=11.203, p=0.0123]$, em 
$\mathrm{T} 1[\mathrm{~F}(1 ; 7)=14.935, \mathrm{p}=0.0062]$, em T3 $[\mathrm{F}(1 ; 7)=7.683, \mathrm{p}=0.0276]$ e em $\mathrm{T} 24[\mathrm{~F}(1 ; 7)=5.846$, $\mathrm{p}=0.0462]$. No grupo CE, a CK foi significativamente superior relativamente a $\mathrm{Tr}$ em $\mathrm{T} 1$

$[\mathrm{F}(1 ; 6)=12.787, \mathrm{p}=0.0117]$ e emT48

$[\mathrm{F}(1 ; 6)=11.088, \mathrm{p}=0.0158]$. Todavia, em T3 o valor da actividade plasmática da CK foi inferior, mas não significativamente $[F(1 ; 6)=5.713, p=0.0540]$.
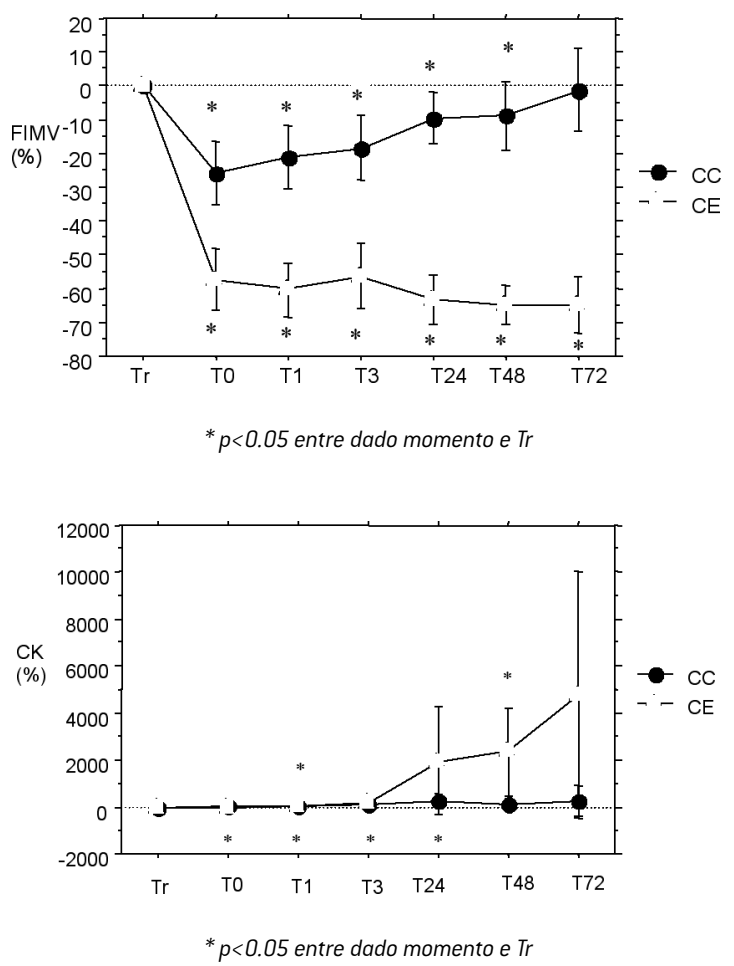

Figura 1. Médias e desvios-padrão da percentagem de variação dos indicadores indirectos de lesão muscular em cada momento de avaliação nos dois grupos estudados.

Legenda: FIMV - força isométrica máxima voluntária; CK - actividade plasmá tica da creatina quinase; CC - grupo de contracções concêntricas; $C \mathcal{E}$ - grupo de contracções excêntricas.

A Figura 2 apresenta as variações dos parâmetros electromiográficos estudados, entre os diferentes momentos de avaliação, em cada grupo.

No grupo CC, a aEMG não apresentou variações estatisticamente significativas ao longo das $72 \mathrm{~h}$. Todavia, verificou-se que os valores foram em todos os momentos superiores a Tr. No grupo que realizou exclusivamente contracções do tipo excêntricas, o panorama é em tudo similar. Contudo, desta feita, em T24 a aEMG foi significativamente superior a $\mathrm{Tr}$ $[\mathrm{F}(1 ; 6)=8.597, \mathrm{p}=0.0262]$. Já em $\mathrm{T} 48$, apesar dos valores de aEMG serem inferiores não existem diferenças significativas $[F(1 ; 6)=5.276, p=0.0613]$. Finalmente relativamente à $\mathrm{MF}$, o grupo $\mathrm{CC}$ apresentou valores significativamente inferiores relativamente a Tr em T0 $[F(1 ; 7)=9.400, p=0.0182]$, T1 $[\mathrm{F}(1 ; 7)=34.068, \mathrm{p}=0.0006], \mathrm{T} 3[\mathrm{~F}(1 ; 7)=24.277$, $\mathrm{p}=0.0017], \mathrm{T} 24[\mathrm{~F}(1 ; 7)=14.554, \mathrm{p}=0.0066]$ e $\mathrm{T} 48$ $[F(1 ; 7)=7.499, p=0.0290]$. No grupo $C E$ os valores foram significativamente inferiores relativamente a Tr em T0 $[F(1 ; 6)=50.840, p=0.0004]$, em T1 $[\mathrm{F}(1 ; 6)=1574.062, \mathrm{p}<0.0001]$, em T3 $[F(1 ; 6)=384.674, p<0.0001]$, em T24 $[\mathrm{F}(1 ; 6)=151.922, \mathrm{p}<0.0001]$, em $\mathrm{T} 48$ $[\mathrm{F}(1 ; 6)=667.436, \mathrm{p}<0.0001]$ e em T72 $[\mathrm{F}(1 ; 6)=14.395, \mathrm{p}=0.0090]$, ou seja, em todos os momentos de avaliação.
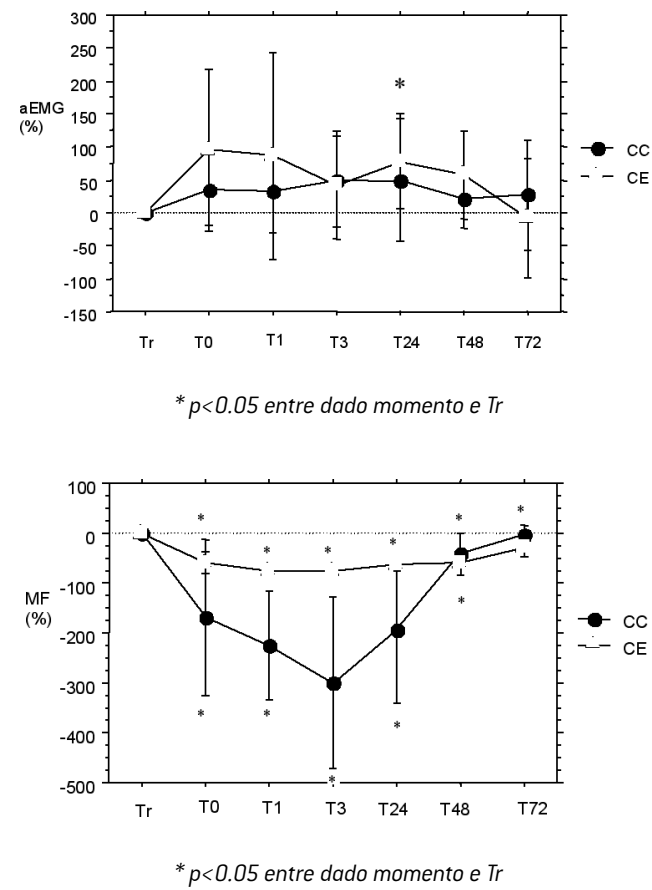

Figura 2. Médias e desvios-padrão da percentagem de variação dos parâmetros electromiográficos em cada momento de avaliação nos dois grupos estudados. Legenda: aEMG - amplitude do sinal electromiográfico; MF - mediana de frequência; CC - grupo de contraç̧ões concêntricas; $C \mathcal{E}$ - grupo de contracções excêntricas. 
Feita a análise da forma como evoluiu cada variável ao longo do tempo em cada grupo de estudo, passou-se de seguida à comparação da variação das mesmas variáveis, mas entre grupos. O Quadro 1 apresenta a percentagem de variação relativamente a $\mathrm{Tr}$ de cada uma das variáveis entre o grupo CC e o grupo CE em cada momento de avaliação.

A variação da FIMV foi significativamente superior no grupo CE relativamente ao grupo CC em todos os momentos de avaliação. No caso da $\mathrm{CK}$, o único momento de avaliação onde se observou uma diferença estatisticamente significativa foi em T48. Às 48 horas, a CK apresentou valores significativamente superiores no grupo CE relativamente ao grupo CC $[\mathrm{t}(6)=-3.115, \mathrm{p}=0.0207]$. No que diz respeito às variáveis electromiográficas, na aEMG não se verificaram diferenças significativas entre os dois grupos em qualquer momento de avaliação. Por fim, a variação da MF foi significativamente superior no grupo CC relativamente ao grupo CE em T1 [t $(6)=-4.599, \mathrm{p}=0.0037]$ , em T3 $[\mathrm{t}(6)=-4.221, \mathrm{p}=0.0056]$ e em T24 [t(6)=2.629, $\mathrm{p}=0.0391]$. No entanto, em T72, os valores foram significativamente inferiores no grupo CC relativamente ao grupo CE $[\mathrm{t}(6)=3.000, \mathrm{p}=0.0240]$.

Quadro 1. Valores médios e respectivos desvios-padrão da percentagem de variação dos indicadores indirectos de lesão muscular e dos parâmetros electromiográficos entre os dois grupos em cada momento de avaliação.

\begin{tabular}{|c|c|c|c|c|c|c|c|c|}
\hline & & $\mathrm{Tr}$ & TO & $\mathrm{T} 1$ & T3 & T24 & $\mathrm{T} 48$ & T72 \\
\hline \multirow{4}{*}{ FIMV } & CC & $0 \pm 0$ & $-25.8 \pm 9.5$ & $-21.3 \pm 9.3$ & $-18.3 \pm 9.7$ & $-9.4 \pm 7.6$ & $-8.9 \pm 10.1$ & $-1.1 \pm 12.2$ \\
\hline & & n.s. & $*$ & * & $*$ & $*$ & * & $*$ \\
\hline & CE & $0 \pm 0$ & $-57.5 \pm 9.1$ & $-60.4 \pm 8.0$ & $-56.3 \pm 9.8$ & $-63.4 \pm 7.2$ & $-64.9 \pm 5.7$ & $-64.9 \pm 8.5$ \\
\hline & CC & $0 \pm 0$ & $26.5 \pm 22.4$ & $51.3 \pm 37.5$ & $101.1 \pm 103.1$ & $245.9 \pm 287.7$ & $144.2 \pm 204.7$ & $252.7 \pm 636.1$ \\
\hline \multirow[t]{3}{*}{ CK } & & n.s. & n.s. & n.s. & n.s. & n.s. & $*$ & n.s. \\
\hline & CE & $0 \pm 0$ & $2.6 \pm 40.0$ & $45.3 \pm 33.5$ & $151.7 \pm 168.0$ & $1952.1 \pm 2303.5$ & $2343.4 \pm 1862.0$ & $4784.5 \pm 5246.2$ \\
\hline & CC & $0 \pm 0$ & $35.4 \pm 53.2$ & $32.3 \pm 61.6$ & $48.6 \pm 69.3$ & $50.0 \pm 92.3$ & $22.1 \pm 45.4$ & $30.7 \pm 82.0$ \\
\hline \multirow[t]{3}{*}{ aEMG } & & n.s. & n.s. & n.s. & n.s. & n.s. & n.s. & n.s. \\
\hline & CE & $0 \pm 0$ & $95.3 \pm 122.7$ & $87.1 \pm 157.5$ & $42.5 \pm 81.5$ & $78.5 \pm 70.8$ & $57.0 \pm 66.4$ & $-8.1 \pm 89.2$ \\
\hline & CC & $0 \pm 0$ & $-168.8 \pm 155.7$ & $-224.6 \pm 108.8$ & $-300.1 \pm 172.3$ & $-194.7 \pm 144.3$ & $-42.0 \pm 43.4$ & $-4.3 \pm 19.3$ \\
\hline \multirow[t]{2}{*}{ MF } & & n.s. & n.s. & $*$ & $*$ & $*$ & n.s. & $*$ \\
\hline & CE & $0 \pm 0$ & $-58.2 \pm 21.6$ & $-73.9 \pm 4.9$ & $-73.6 \pm 9.9$ & $-61.1 \pm 13.1$ & $-60.8 \pm 6.2$ & $-28.4 \pm 19.8$ \\
\hline
\end{tabular}

Legenda: FIMV - força isométrica máxima voluntária; CK - actividade plasmática da creatina quinase; aEMG - amplitude do sinal electromiográfico; MF - mediana de frequência; CC - grupo de contracções concêntricas; CE - grupo de contracções excêntricas; * $p<0.05$.

\section{DISCUSSÃO E CONCLUSÕES}

A FIMV e a CK são duas das variáveis fisiológicas mais utilizadas enquanto indicadores indirectos da lesão induzida por cargas exaustivas e/ou inabituais. Assim, as referidas variáveis foram estudadas no presente trabalho no sentido de se poder averiguar se a carga aplicada aos sujeitos induziu lesão muscular. A FIMV apresentou, nos dois grupos, reduções significativas em todos os momentos de avaliação, em comparação com Tr, excepto entre Tr e T72 no grupo CC. Na verdade, diversos estudos também constataram que ocorriam diminuições acentuadas na FIMV após a aplicação de protocolos exaustivos e/ou inabituais. No entanto, essas diminuições eram mais acentuadas ao realizar-se exercícios do tipo excêntrico do que do tipo concêntrico $(3,10,18,34)$. A justificação para esta redução da FIMV será motivada por dois factores. Ela poderá resultar de alterações metabólicas, imediatamente após a aplicação do exercício; ou pode ser devida à lesão muscular que foi induzida (3). No entanto, a questão que se coloca do ponto de vista experimental é quando existirá claramente um predomínio dos factores associados à fadiga ou à lesão muscular (3). 
A CK, no grupo CC, apresentou relativamente a $\mathrm{Tr}$ valores significativamente superiores em $\mathrm{T} 0$, em $\mathrm{T} 1$, em T3 e em T24. Já no grupo CE ela foi significativamente superior em $\mathrm{T} 1$ e em $\mathrm{T} 48$ do que em $\mathrm{Tr}$. Ou seja, parece que tal como já fora descrito na literatura, também neste caso se verificou uma libertação tardia da CK para a corrente sanguínea $(2,8)$. Assim, no presente estudo o pico da CK para o grupo CC aconteceu às 24 horas e para o grupo CE às 72 horas, não obstante o último resultado com valores significativos ter ocorrido às 48 horas. A justificação para tal fenómeno reside no transporte da CK que se realiza fundamentalmente pela circulação linfática, mais lenta do que a circulação sanguínea $(8,26)$. Olhando atentamente para os desvios-padrão nos diferentes momentos, em ambos os grupos, observam-se valores razoavelmente elevados. Clarkson et al. (5) argumentam que isso se deverá à capacidade de alguns sujeitos removerem mais rapidamente a CK do que outros. Isto é, parece que existirá uma forte variabilidade interindividual na capacidade de remoção da CK.

Resumindo, a partir da análise destes dois indicadores indirectos, os sujeitos do grupo CC e do grupo $\mathrm{CE}$ apresentavam fortes evidências de que se encontravam com uma lesão muscular induzida pelo exercício exaustivo e inabitual. Assim sendo, o passo seguinte constituiu em conhecer as possíveis repercussões dessa patologia a nível neuromuscular. Em termos genéricos não se observaram diferenças significativas da aEMG, quer no grupo CC, quer no grupo $\mathrm{CE}$ ao comparar os diferentes momentos de avaliação com Tr. Linnamo et al. (17), McHugh et al. (21), Saxton e Donnelly (32) e Sbriccoli et al. (33) estudaram parâmetros electromiográficos no domínio temporal e também não verificaram variações significativas. Todavia, há que frisar que se observaram aumentos desta variável, não obstante não terem qualquer significado estatístico.

Hipoteticamente este aumento da actividade neuromuscular surgirá para atenuar a redução da resposta mecânica do músculo $(15,21,30,31)$. Ou seja, isto sugere um ligeiro aumento no nível de recrutamento para compensar uma diminuição da produção de força. Assim, o problema não residirá no mecanismo de recrutamento de unidades motoras, mas sim na falha ou redução de actividade no mecanismo de contracção (33).

No grupo CC, verificou-se que a MF apresentou relativamente a $\operatorname{Tr}$ valores significativamente inferiores em todos os momentos excepto em T72. No grupo $\mathrm{CE}$ os valores foram significativamente inferiores em todos os momentos de avaliação. O comportamento desta variável parece que não é consensual. De facto, McHugh et al. (21), Saxton e Donnelly (32) não observaram diferenças significativas na MF entre os diversos momentos de avaliação. Por outro lado, um outro conjunto de autores $(6,17$, 33) verificaram diminuições significativas da MF nos diversos momentos de avaliação com os valores antes da aplicação do protocolo, em sujeitos que realizaram quer tensões do tipo concêntrico, quer do tipo excêntrico. Assim sendo, os resultados do presente estudo parecem estar em congruência com os descritos pelos últimos autores citados. Esta redução da MF significa um aumento das componentes de baixas frequências e uma diminuição das componentes de altas frequências. Este facto poderá decorrer de diversos factores, entre os quais o padrão de recrutamento sincronizado das unidades motoras, a diminuição da velocidade de condução do potencial de acção, ou a passagem de dominância de recrutamento de fibras tipo II para fibras tipo I (29). Por outras palavras, devido ao tipo de exercício aplicado no protocolo ter uma solicitação predominante de fibras tipo II, estas poderão ser as mais lesadas, levando após o protocolo a que ocorra um recrutamento fundamentalmente de fibras tipo I $(9,17)$. Comparando o grupo CC e o grupo CE em cada momento de avaliação, verificou-se que a FIMV foi significativamente superior no grupo $\mathrm{CE}$ em todos os momentos de avaliação excepto em Tr. Com efeito, os resultados do presente estudo parecem ser similares aos de Monteiro (24). No grupo CE observaram-se dois momentos de diminuição da FIMV. Um primeiro logo após o exercício e um segundo às 24 horas, como já tinha sido descrito (18). Num primeiro momento a explicação para este fenómeno residirá nos factores metabólicos associados a factores de lesão mecânica. Já num segundo momento a explicação para a diminuição da FIMV poderá residir nos mecanismos de reacção inflamatória tecidual. 
No caso da CK, o único momento de avaliação onde se observou uma diferença estatisticamente significativa entre os grupos foi às 48 horas. No entanto, a partir de T3, a CK foi sempre superior no grupo CE do que no grupo CC. A explicação para não se verificarem diferenças significativas, particularmente às 24 e às 72 horas, poderá dever-se à elevada variabilidade inter-individual, expressa pelos elevados valores de desvio-padrão. Assim, parece evidente que ambos os exercícios são manifestamente lesivos, mas com um maior grau no caso das cargas excêntricas.

Não se observaram diferenças significativas entre os dois grupos em qualquer momento de avaliação na aEMG. Na verdade, Sbriccoli et al. (33) também não encontraram diferenças significativas em qualquer momento de avaliação. O que indicará que não existiram diferenças significativas no recrutamento de fibras, independentemente do tipo de protocolo utilizado. A MF foi significativamente superior no grupo CC do que no grupo CE em T1, em T3 e em T24.

Todavia, este facto não parece estar em concordância com a literatura, onde frequentemente se verifica que a MF apresenta maiores diminuições nos exercícios excêntricos do que nos concêntricos (17). No entanto, em T72, os valores do grupo CC foram significativamente inferiores aos do grupo CE. Isto poderá significar que às 72 horas, pelo menos relativamente à actividade neuromuscular, já existe um restabelecimento completo da lesão quando se efectuam exercícios do tipo concêntrico. No entanto, o mesmo já não se pode afirmar relativamente a cargas do tipo excêntrico, podendo ocorrer que o processo de recuperação passe para lá desse meato temporal. Em síntese, a realização do exercício excêntrico implementado no presente estudo, com um carácter inabitual e exaustivo, induz maiores evidências de lesão muscular comparativamente ao exercício concêntrico. Essa lesão parece ser selectiva, afectando particularmente as fibras do tipo II, o que é expresso pela diminuição da MF.

\author{
CORRESPONDÊNCIA \\ Tiago Barbosa \\ Instituto Politécnico de Bragança \\ Departamento de Ciências do Desporto e \\ Educação Física \\ Campus de Sta. Apolónia \\ Apartado 1101 \\ 5301-856 Bragança, Portugal \\ barbosa@ipb.pt
}




\section{REFERÊNCIAS}

1 Appel, J.; Soares, J. \& Duarte, J. (1992). Exercise, muscle damage and fatigue. Sports Med., 13: 108-115.

2 Armstrong, R. (1990). Initial events in exercise-induced muscular injury. Med. Sci. Sports Exerc, 22: 429-435.

3 Armstrong, R.; Warren, G. \& Warren, J. (1991). Mechanisms of exercise-indiced muscle fibre injury. Sports Med., 12:184-207.

4 Clarkson, P.; Byrnes, W.; McCormick, K.; Turcottr, L. \& White, J. (1986). Muscle sorness and serum creatine kinase activity following isometric, eccentric and concentric exercise. Int. J. Sports Med., 7: 152-155.

5 Clarkson, P.; Nosaka, K \& Braun, B. (1992). Muscle function after exercise-induced muscle damage and rapid adaptation. Med. Sci. Sports Exerc., 24, 512-520.

6 Day, S.; Donnelly, A.; Brown, S. \& Child, R. (1998). Electromyogram activity and mean power frequency in exercise-damage human muscle. Muscle and Nerve, 21: 961-963.

7 Duarte, J. (1993). Lesões celulares do músculo esquelético induzidas pelo exercício físico. Tese de Doutoramento. Porto: Faculdade de Ciências do Desporto e de Educação Física da Universidade do Porto.

8 Ebbeling, C \& Clarkson, P. (1989). Exercise-induced muscle damage and adaptation. Sports Med., 7: 207-234.

9 Enoka, R. (1988). Neuromechanical basis of Kinesiology. Champaign, Illinois: Human Kinetics.

10 Faulkner, J. Brooks, S. \& Opiteck, J. (1993). Injury to skeletal muscle fibres during contractions: conditions of accurence and prevention. Phys. Ther., 73: 911-921.

11 Foley, J.; Jayaraman, R.; Prior, B.; Pivarnik, J. \& Meyer, R. (1999). MR measurements of muscle damage and adaptation after eccentric exercise. J. Appl. Pysiol., 87: 2311-2318.

12 Fridén, J.; Sfakianos, P. \& Hargen, A. (1986). Muscle sorness and intramuscular fluid pressure: comparison between eccentric and concentric load. J. Appl. Physiol., 6:2175-2179.

13 Hakkinen, K. \& Komi, P. (1983). Electromyographic changes during strength training and detraining. Med. Sci. Sports Exerc., 15: 455-460.

14 Hakkinen, K.; Komi, P.; Alén, M. \& Kauhanen, H. (1988). EMG, muscle fibre and force production characteristics during a 1 year training period in elite weight-lifters. Eur. J. Appl. Physiol., 56: 419-427.

15 Kent-Braun, J. (1999). Central and peripherical contributions to muscle fatigue in humans during sustained maximal effort. Eur. J. Appl. Physiol., 80: 57-63.

16 Lieber, R.; Fridén, J. (1993). Muscle damage is not a function of muscle force but active muscle strain. J. Appl. Physiol., 74: 520-526.

17 Linnamo, V.; Bottas, R. \& Komi, P.V. (2000). Force and EMG power spectrum during and after eccentric and concentric fatigue. J. Electromyography and Kinesiology, 10: 292-300.

18 MacIntyre, D.L., Reid, W.D., Lyster, D.M., Szasz, I.J., \& McKenzie, D.C. (1996). Presence of WBC, decreased strength, and delayed soreness in muscle after eccentric exercise. J. Appl. Physiol., 80: 1006-1013.

19 Magalhães, J. (1996). Exercícios físicos inabituais e exaustivos em crianças. Influência do tipo predominante de contracções em indicadores indirectos de stress oxidativo e de agressão/lesão muscular esquelética. Tese de Mestrado.
Porto: Faculdade de Ciências do Desporto e de Educação Física da Universidade do Porto.

20 Magalhães, P. (2000). Alterações hematológicas agudas induzidas por diferentes protocolos de exercício físico exaustivo e inabitual. Tese de Mestrado. Porto: Faculdade de Ciências do Desporto e de Educação Física da Universidade do Porto.

21 McHugh, M., Connolly, D., Eston, R., \& Gleim, G. (2000). Electromyographic analysis of exercise resulting in symptoms of muscle damage. J. Sports Science, 18: 163-172.

22 Moritani, T; Muramatsu, S \& Muro, M. (1988). Activity of motor units during concentric and eccentric contractions. Am. J. Phy. Med., 66: 338-350.

23 Mota, P. (1995). Estudo comparativo dos indicadores indirectos de lesão muscular induzidas pelo exercício em crianças e adultos. Tese de Mestrado. Porto: Faculdade de Ciências do Desporto e de Educação Física da Universidade do Porto.

24 Monteiro, L. (1996). Agressão/lesão muscular esquelética em crianças induzida pelo exercício físico. Influência do tipo predominante de contracções na manifestação de diferentes indicadores indirectos. Tese de Mestrado. Porto: Faculdade de Ciências do Desporto e de Educação Física da Universidade do Porto.

25 Nakazawa, K.; Kawakami, Y.; Fukunaga, T.; Yano, H. \& Miyashita, M. (1993). Differences in activation patterns in elbow flexor muscles during isometric, concentric and eccentric contractions. Eur. J. Appl. Physiol., 66: 214-220.

26 Newham, D.J., Jones, D.A., \& Clarkson, P.M. (1987). Repeated high-force eccentric exercise: effects on muscle pain and damage. J. Appl. Physiol.: 63, 1381-1386.

27 Nosaka, K \& Newton, M. (2001). Force deficit immediately post eccentric exercise predicts the magnitude of muscle damage. In: J. Mester, G. King, H. Struder, E. Tsolakidis \& A. Osterburg (eds.). Book of Abstracts of the 6th Annual Congress of the European College of Sport Science. Cologne: Sport und Buch Strauss, 76.

28 Rodenburg, J.; Deboer, R.; Schiereck, P.; van Echteld, C. \& Bar, P. (1994). Changes in phosphorus compounds and water content in skeletal muscle due to eccentric exercise. Eur. J. Appl. Physiol., 68: 205-213.

29 Pezarat Correia, P., Mil-Homens, P., \& Veloso, A. (1993). Electromiografia. Fundamentação fisiológica. Métodos de recolha e processamento. Aplicações cinesiológicas. Lisboa: Edições da Faculdade de Motricidade Humana.

30 Potvin, J. (1997). Effects of muscle kinematics on surface EMG amplitude and frequency during fatiguing dynamic contractions. J. Appl. Physiol., 82: 144-151.

31 Potvin, J., \& Bent, L. (1997). A validation of techinques using surface EMG signals from dynamic contractions to quantify muscle fatigue during repetitive tasks. J. Electromyogr. Kinesiol., 7: 131-139.

32 Saxton, J. \& Donnelly, A. (1996). Length-specific impairement of skeletal muscle contractile function after eccentric muscle actions in man. Clinical Sci., 90: 119-125.

33 Sbriccoli, P.; Felici, F.; Rosponi, A.; Aliotta, A.; Castellano, V.; Mazzà, C.; Bernardi, M. \& Marchetti, M. (2001). Exercise induced muscle damage and recovery assessed by means of linear and non-linear sEMG analysis and ultrasonography. J. Electromyography and Kinesiology, 11: 73-83. 
34 Soares, J.; Duarte, J. (1989). Miopatia do exercício.

Etiologia, fisiopatologia e factores de prevenção. Rev. Port. Med. Desp., 7: 187-200.

35 Soares, J.; Mota, P.; Duarte, J. \& Appell, H.J. (1996).

Children are less susceptible to exercise-induced muscle damage than adults: a preleminary investigation. Pediat.

Exerc. Sci., 8: 361-367. 